\title{
The Focus-Metaphor Approach: A Novel Concept for the Design of Adaptive and User-Centric Interfaces
}

\author{
Sven Laqua ${ }^{1}$ and Paul Brna ${ }^{2}$ \\ ${ }^{1}$ Northumbria University, Newcastle upon Tyne \\ slaqualsl-works. de \\ ${ }^{2}$ University of Glasgow \\ paul.brna@scre.ac.uk
}

\begin{abstract}
The Focus-Metaphor Approach is a novel concept for the design of adaptive and user-centric virtual environments which seeks to use a form of associativity to adapt the interface to the user whilst keeping one primary focus element and many secondary and peripheral focus elements. In this paper, the underlying theory is presented and differentiated from related research. The proposed solution has been implemented as a prototype and tested for usability issues using an online evaluation and in-laboratory eye-tracking to find some evidence that time spent off-communication is reduced. The results are reported briefly, implications considered and the areas for further work are pointed out.
\end{abstract}

\section{Introduction}

Collaborative systems require the integration of facilities for a wide range of activities including chatting, exchanging emails, working with a shared application and finding resources for both personal and shared use.

The use of many of these tools involves significant time away from what is often the central focus - communicating effortlessly with others. For example, sending an email address to someone through a chat interface might require firing up an email program and searching through a set of email addresses, then copying and pasting this into a chat box. Even where applications are smarter, many times there is much "off communication" activity.

This raises the issue of whether GUI interfaces could be "smarter", responding to contextual clues without any specific direction from the learner. Can the time spent in navigating the interface be usefully reduced? One possible solution is presented here the "Focus-Metaphor approach", which seeks to use a form of associativity to adapt the interface to the user whilst keeping one primary focus element, and many secondary and peripheral focus elements. The proposed solution has been implemented and tested for usability issues and to find some evidence that time spent off-communication is reduced. The results are reported briefly and implications considered.

\subsection{Related Work}

Interfaces that are more usable, learnable and satisfying may allow people to concentrate on establishing good relationships with each other across the net, freeing them 
up from some of the tedious navigation tasks to concentrate, for example, on establishing a trusting relationship with other learners or teachers.

Various other researchers have proposed aids to assist navigating through a large information space. In several articles, Card et al. [1+2] describe their research on Degree of Interest (DOI) Trees, aimed to browse through large hierarchies of information. Using a similar approach, TheBrain [3] has developed solutions called PersonalBrain, Web-Brain and BrainEKP (Enterprise Knowledge Platform).

But as these solutions only focus on the visualization of navigation structures the problem which still remains in their approaches is the separation of navigation and information representation. This separation causes abrupt switches between navigation and information, which especially disrupts cognitive processes. The Focus-Metaphor approach aims to overcome this issue to support user orientation and facilitate learning processes.

Moreover, there are examples which make use of the so called fish-eye view technique, like described in work by Gutwin [4] or Thomas [5], the fishnet browser [6] or in a modified form to be seen in the ICQ universe [7]. These fish-eye view implementations allow preserving "the contextual relationship between a large number of objects" [8]. But although the fish-eye view technique shows visual similarities to the Focus-Metaphor approach, there is still a wide difference in the underlying concept. Whereas the fish-eye view tries to provide a visually appealing solution for navigating large amounts of static information, the approach taken here is different - i.e. to reorganise the information space into a focus, secondary foci and peripheral foci, hiding the rest of the space. Doing this in a contextually sensitive manner may well do the majority of the work for the learner so that they can concentrate on their communication with others. Using "cognitive modelling" [9] to organise the information space and using the spreading activation approach to assign information to according foci would be one solution to achieve this goal of an intelligent interface using the FocusMetaphor approach.

\section{The Theory}

The Focus-Metaphor approach combines aspects of design theory, cognition psychology and educational theory to create a more natural way of interaction. With the design of the Focus-Metaphor it was intended to achieve advancements over commonly used metaphors (e.g. desktop metaphor, portal metaphor) and counteract effects like "second visit blindness on websites" [10]. This approach uses the principle of cortical connectivity and its importance for cognitive functions [11] to apply them to the design of dynamic and adaptable user interfaces.

\subsection{The Connection Between Navigation and Learning Processes}

Using hypertext environments like the Internet for the development of learning applications requires an understanding of the importance of navigation, which is more than just the way of orientation and interaction [12]. It is especially an active form of learning, where the way people navigate from one information entity to the next directly influences the way they process this information and possibly acquire new 
knowledge. Along with Fuster [11], "learning and the acquisition of memory are based on the synaptic linkage of elementary cortical representations".

Everyone has an individual network of personal knowledge which he or she tries to expand through interaction with new information. This individual mental network consists of countless nodes of information entities, organized in topological, nonlinear structures [13]. When interacting with virtual spaces like hypertext environments, the mental network of knowledge of an individual is confronted with the virtual network of information of the according environment.

Whereas novel educational research focuses on constructivism [14] and related methods like "experimental learning" [15] or "discovery learning" [14], under the assumption that every individual has a different way to acquire knowledge, information in virtual spaces is mainly static. Of course hyperlinks can provide some amount of flexibility if used in a sensible way, but their advantages are increasingly undermined by overloaded or badly structured screens. This leads to a virtual network of information which forces people to adapt their way of thinking to these static structures. Actual research on semantic web technologies underlines the existence and importance of this problem and tries to give more meaning to information.

The concept of the Focus-Metaphor suggests the design of a dynamic and highly adaptable interface to reduce the gap between people's individual network of knowledge and the presentation of information on the screen. A Focus-Metaphor interface (FMI) bases its visual representation on cognition psychological principles and aims to support learning processes.

\subsection{The Hierarchical Structure of a Focus-Metaphor Interface (FMI)}

A hierarchical structure is used to manage the visualized elements. The number of these elements should be around five to seven, according to the capacity of short-term memory [16]. “...instructional design for the learning purpose should keep the limits of short-term memory in mind" [17].

In a Focus-Metaphor interface, the element of most interest for the user - the "primary focus element" [18] - is presented in the middle of the screen having the largest dimensions (see fig. 1). Other relevant elements, which are also of interest for the user and which are in context to the 'focused' element, are dynamically grouped and arranged around the primary focus element. These elements represent the "secondary focus". The third hierarchy is called the "peripheral focus" and shows elements which may not be in context of the primary focus element, but still are essential for a fluent and effective interaction of the user with the system (e.g. functions like search, news, contact).

\subsection{The Level of Detail of Information}

A comparison of the Focus-Metaphor approach to existing but novel user interfaces like the grid- or table-layout approach which are used by myNetscape [19], myMSN [20] and others, reveals a core difference: These approaches allow the user to customize the presented information by arranging and showing or hiding information 'modules', but they are still static and plain two-dimensional, without contextual intelligence. 
The wide difference of the Focus-Metaphor approach is the usage of hierarchical information and its hierarchical representation, which should be managed through "information importance level" [18] and the allocation to the according presentation level. Depending on the level of presentation, the amount of presented information in each element is varying.

Only this combination of hierarchical organization of information, dynamically allocated and animated in real-time, as basis for a visual hierarchy, can reasonably enable the Focus-Metaphor approach. This hierarchical order shall help learners to concentrate on one specific and important context and thereby support learning processes. In addition, to limit the amount of information presented to the user through the visual elements in the different importance level, information which is not necessary disappears.

\subsection{How the Focus-Metaphor Works}

If the user switches his or her focus from the element of primary focus onto an element of secondary or peripheral focus, the interface reacts in an appropriate manner. In the prototype of this project, the described interaction has been realized through mouse-over events, but in future developments an interface implementation might also use eye-tracking data and fixation measurements for the navigation.

Independent of which form of interaction is used, the user needs to 'focus' on an element, which then comes to the front (technically: switches to the top layer) and provides its information completely. This is necessary, as through the possible and likely overlapping of elements of the different foci, the view onto some elements might be restricted. If the user decides to stay with his focus on the new element, he or she 'selects' this element. This has been realized in the prototype via mouse-click, but alternatively an eye-tracking interface could use longer fixations or eye-blink for this interaction. Then, the chosen element takes over the primary focus, presenting its complete information. This forces the other elements to rearrange according to their relation with the new primary focus element. Some presented elements might therefore disappear and others show up. As a result, the user can experience a feeling of involvement and of active interaction where he seems to be a navigator in a networked world of knowledge nodes. As the screenshot of the interface prototype in fig. 1 on the next page shows, the presented elements are specific modules of information, which together build the learning environment. Smooth and continuous motions shall be used to switch focus and rearrange elements.

\section{Prototype}

In order to evaluate the usability of the Focus-Metaphor Approach, a narrow but highlevel prototype, which uses a collaborative story-writing context, has been implemented, tested and evaluated. This prototype has been designed as a virtual, webbased and therefore distributed environment which consists of a back-end using Java and MySQL and a front-end using Macromedia Flash. Within the scope of this project, attention focused on the testing of fundamental usability issues and the general user acceptance in a realistic scenario using a formative online evaluation, experts and 
as core element an in-laboratory testing with an SMI iViewX eye-tracking system. For the evaluation, test users were instructed to write personal stories. These have been used to find similarities among the contributors communicate these similarities and facilitate interaction within the hopefully emerging community. Fig.1 below shows a screenshot of the prototype, which models the elements of primary and secondary focus. (Also see: http://www.fmi.laqua-consulting.de/Test-Area.html)

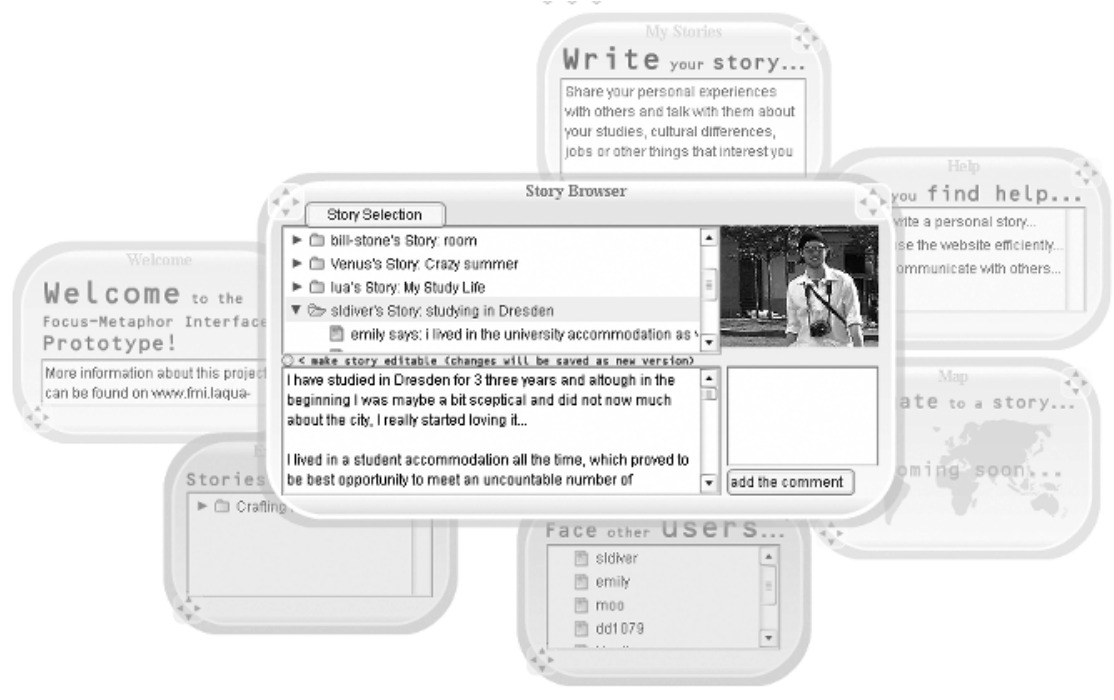

Fig. 1. Screenshot of the Focus-Metaphor Interface prototype

\subsection{Modules}

For the Focus-Metaphor Interface prototype, nine different modules (also referred to as elements in a specific focus mode) have been developed altogether in order to enable realistic testing scenarios.

The Welcome Module provides some general information about the project. It contains a link to the main project website and furthermore gives a brief description of how the online testing works and what the aims are for the user.

In the Registration Module, every user who wants to test the FMI prototype needs to fill in the registration form. A nickname and a password are required in order to login. All other entries can be filled in on a voluntary basis.

After a successful registration, the test user can log into the website using the Login Module. Nickname and password are required to gain access and thereafter, the user is automatically directed to the FMI testing environment.

With the Help Module, the test user can access some guidance on how to work with the FMI prototype. This information is stored in a separate XML file on the webserver, which makes it easy to administrate changes or extensions.

The MyStories Module allows the test user to write a personal story about him- or herself including a title and a location in plain text style. 
The StoryBrowser Module is used to provide an overview on all written stories on which test users can write comments on. A picture of every story- or commentauthor is displayed and the test user can optionally choose to display just the five newest stories or his or her stories only.

The Map Module visualizes the locations of written stories to provide test users a better feeling for the community.

In the Expert's Stories Module information from a professional story-teller [21] has been integrated. It provides additional guidance for the user on how to write a good personal story and what it is useful for.

The User's Module lists all registered users and provides their names, locations and e-mails, according to the information which has been provided with the registration. For each test user who agreed and provided a picture, this has been uploaded onto the server and is also displayed in the user's module.

\section{Online Evaluation}

The formative online evaluation included a usability questionnaire [22] basing on Lewis [23] work: "IBM Computer Usability Satisfaction Questionnaires: Psychometric Evaluation and Instructions for Use." The questionnaire consisted of nineteen statements, to be marked between 1 (strongly disagree) and 6 (strongly agree) and has been completed by fifteen participants, focusing on their personal opinion, feelings and satisfaction with the Focus-Metaphor interface prototype. Reasoned by the novelty and, in comparison with usual web interfaces, dissimilarity of the FocusMetaphor approach, feedback about the participant's emotions and attitude when using the prototype was the core aspect of this investigation.

The results of this first usability evaluation of a Focus-Metaphor realization showed that the overall feedback on the prototype has been very satisfying. All statements were marked positive (above 3.5), with an overall geometric mean of 4.63 (arithmetic mean $=4.64$; harmonic mean $=4.62$ and median $=4.68$ ).

Nevertheless, it might be argued that the setting of the evaluation environment caused a shift towards more positive answers due to the fact that the formative part of the evaluation also involved quite some communication flow between participants and investigator. To cope with this, the analysis focused on the high and low peaks of the feedback, which are clearly above or below any of the various means or the median, and therefore ensure reliability of the results.

Table 1. Ranking of the online evaluation feedback

\begin{tabular}{lll}
\hline Highest & 5.05 & The information provided for the system is easy to understand \\
& 4.87 & I can effectively complete my work using this system \\
4.87 & It was simple to use this system \\
4.86 & The organization of information on the system screens is clear \\
& & \\
& 4.31 & Whenever I make a mistake using the system, I recover easily and quickly \\
& 3.97 & This system has all the functions and capabilities I expect it to have \\
Lowest & 3.94 & The system gives error messages that clearly tell me how to fix problems \\
\hline
\end{tabular}


The ranking of feedback given on the statements (see table 1 above) shows the highest and lowest means according to a specific statement. The positive results show that the novelty of the interface has not been an issue for the participants. But the negative results also show that there are still serious issues which can be explained by the very narrow prototype which does not raise any claim to be a finished product. The mentioned problems are important, but obviously would be removed when developing a complete environment using the Focus-Metaphor approach.

Moreover, the online prototype has been developed with two interface options, an 'automated animation' version and a manual version. Whereas the first version provided fixed locations for the modules in primary and secondary focus mode and continuous animation, the second version allowed participants to arrange the modules freely on the browser screen. By voting on these two versions, the participants expressed their preference for the animated version, which as a result has been chosen as the central representation mode for the Focus-Metaphor interface prototype.

In addition, this mode has been chosen to be the fundament for the eye-tracking experiment.

\section{$5 \quad$ Eye-Tracking Experiment}

The overall aim of the eye-tracking experiment was to compare an interface using the Focus-Metaphor approach with a common interface which has a static layout like most websites. To have comparable results, the same visual information (factor $\mathrm{X}$ ) needed to be presented to the participants during the experiment. Accordingly, three different derivatives have been created from the original prototype version of the Focus-Metaphor interface:

$\mathrm{X}[1]$ : The original online version (Focus-Metaphor): animated with secondary focus modules centered around the primary focus module

$\mathrm{X}[2]$ : The adapted static version (grid layout): based on common website structures, using a grid layout and the same information as X[1],

$\mathrm{X}[3]$ : The adapted original version (Focus-Metaphor): similar to X[1] but without animation.

The researched context within the eye-tracking experiment for the versions $X[1]$, $\mathrm{X}[2]$ and $\mathrm{X}[3]$ was to measure the participants' visual attention (behaviour $\mathrm{Y}$ ) onto the primary focus module in the middle of the screen, which always provides the 'actual' content.

With the investigation of how X[1], X[2] and X[3] affect Y, the hypothesis "the focus-metaphor improves learning processes in the researched context" has been tested indirectly through the two assumptions: (1) A better (visual) focus on the learning context and (2) an optimized orientation.

For the conduction of the eye-tracking experiment, a population sample of fifteen participants has been used. All participants worked with the real prototypes (X[1], $\mathrm{X}[2]$ and $\mathrm{X}[3]$ ) rather than in predefined paths to allow higher realism of the experiment.

Before the experiment started, participants had been given a scenario form which briefly described the interactions they should undertake whilst conducting the ex- 
periment. Along with the three different versions of the prototype (X[1], X[2] and $\mathrm{X}[3])$ the experiment was separated into three sessions. In each session, the participant dealt with one of the prototype versions for five to seven minutes, depending on how quick the participant progressed within the given scenario. The setting of the experiment aimed to allow the participants to explore and work with the prototype in a natural way. The only interfering element has been a chin-rest to improve the quality of the gathered data. The IView X System by SMI, which has been used for the conduction of this experiment, did not require any further disturbing parts like headmounted elements.

In addition to the recording of the eye-tracking data (gaze path, pupil diameter and fixations), the participant's screen was video-captured in every session and overlaid with the participant's gaze. This method led to 3.5 hours of video data, which was analyzed to define typical interaction sequences like working with the story-browser module, the user's module, the help module, the expert's stories module or the welcome module. These sequences have then been used for the further analysis with the IView X Analysis software.

\subsection{Overall Attention Analysis}

The overall attention analysis compared the eye-tracking data of the different versions $\mathrm{X}[1], \mathrm{X}[2]$ and $\mathrm{X}[3]$ for the length of a complete session (without the login phase) in order to measure the overall visual attention of participants (Y_all) on the primary focus module. Therefore Y1_all, Y2_all and Y3_all represent the time, which test users in average spend on the primary focus module in each version of the prototype from a successful login until the end of the testing session in percent of the length of the whole session (minus login time). The higher this percentage, the longer test users have dealt with the main content, which they did choose on their own during the session. This measure can provide clues about the effectiveness of the interface versions. The values of Y1_all, Y2_all and Y3_all allow conclusions about how long users needed for navigation tasks. The more time a user spent on the actual content, the less time he required for navigating through the prototype environment. In reverse, less time spent on the content indicates more time consuming navigation, which therefore disturbs the user from studying the desired content.

As expected, the individual results of the participants showed strong variations, but of course the investigation focused on the quantified means of each version. Here, the comparison of X[1] and X[3] (both are Focus-Metaphor Interfaces) shows with the geometric means Y1_all $=\mathbf{7 5 . 9} \%$ \& Y3_all $=\mathbf{7 5 . 7} \%$ basically identical values . The standard error $\sigma_{\mathrm{M}}$ for both means, Y1_all and Y3_all, is $\boldsymbol{\sigma}_{\mathbf{Y} 1 \_ \text {all }}=\boldsymbol{\sigma}_{\mathbf{Y} 3 \_ \text {all }}=\mathbf{1 . 8}$. This led to the conclusion that the omission of the animation did not affect the visual attention. Moreover, the result underlines the reliability of the conducted experiment, as the very similar versions deliver nearly the same values. In contrast, the analysis of $\mathrm{X}[2]$ delivered with a geometric mean of Y2_all $=\mathbf{6 4 . 1} \%$ a clearly lower value. The standard error shows a similar result with $\boldsymbol{\sigma}_{\mathbf{Y} 2 \_ \text {all }}=\mathbf{3 . 7}$. The relatively high increase of overall visual attention when using the Focus-Metaphor Interface (11.6\% for Y3_all and $11.8 \%$ for Y1_all) instead of the standard grid layout interface is a first indication for an increased attention on the 'learning context' due to less time spent on navigation tasks. 
Therefore, the overall attention analysis supports the first assumption of the hypothesis of providing a better focus onto the learning context. Moreover, with the correlation of $\mathrm{Y} 1$ and $\mathrm{Y} 3$ and time constraints within the project, this led to the decision to investigate only $\mathrm{X}[1]$ and $\mathrm{X}[2]$ for the further analyses (see fig. 2).
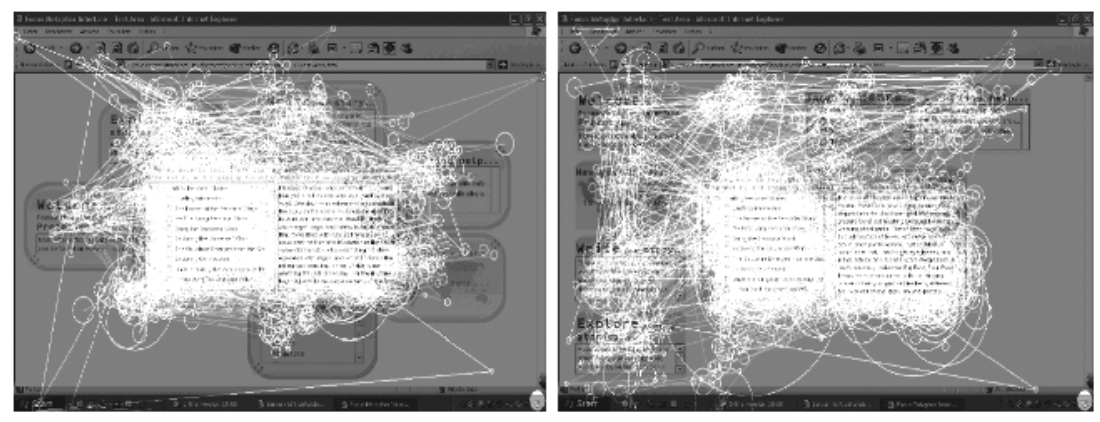

Fig. 2. Visualization of Gaze Paths of all Eye-Tracking Participants for Focus-Metaphor Interface X[1] (left) and Grid-Layout Interface X[2] (right) for the overall session

\subsection{Study Phase Analysis}

The study phase analysis aimed to find differences in the participants' visual focus on the learning context while working with the versions X[1] and X[2]. In contrast to the prior overall attention analysis, the study phase analysis refers only to the time, in which the participants worked with a specific module. This means, that the small periods of "loading" new content have been neglected. Only the time, which a user spent on the modules in primary focus mode to read stories or comments, study information in general or watch pictures, has been considered. As some modules did not provide enough information to study them for a longer period, only relevant modules had been included in the investigation. These modules were the welcome module, the help module, the story browser module, the user browser module and the expert's stories module, which contained most of the content and also showed average the longest study times. The results of the study phase analysis show that the visual attention in $\mathrm{X}[1]$ is noticeably higher than in X[2] (see geometric means for the different modules, according standard errors and confidence intervals in table 2).

Table 2. Comparison of attention on the according moduls during study phases

\begin{tabular}{c|rrr|rrr}
\hline \multirow{2}{*}{ modules } & \multicolumn{3}{|c|}{ Focus-Metaphor } & \multicolumn{3}{c}{ Grid Layout } \\
& in \% & $\boldsymbol{\sigma}_{\mathbf{Y} \mathbf{1}}$ & Conf. Interval & in \% & $\boldsymbol{\sigma}_{\mathbf{Y 2}}$ & Conf. Interval \\
\hline Welcome & 79.3 & 1.9 & $71,3 \leq \mu \leq 87,3$ & 49.4 & 4.5 & $35,6 \leq \mu \leq 64,3$ \\
Help & 80.6 & 2.4 & $75,1 \leq \mu \leq 86,0$ & 73.4 & 3.0 & $66,6 \leq \mu \leq 80,2$ \\
Story browser & 84.9 & 1.1 & $82,7 \leq \mu \leq 87,2$ & 73.8 & 2.4 & $68,8 \leq \mu \leq 78,7$ \\
User browser & 79.0 & 3.2 & $70,6 \leq \mu \leq 87,3$ & 63.1 & 7.5 & $46,2 \leq \mu \leq 80,1$ \\
Expert stories & 79.4 & 2.5 & $73,5 \leq \mu \leq 85,2$ & 74.3 & 3.2 & $66,4 \leq \mu \leq 82,2$ \\
\hline
\end{tabular}


These means, for the modules which have been studied by the participants, show that the Focus-Metaphor Interface delivered higher results in every module. But as the results for the separate modules show relatively high variations (see the confidence intervals in table 2), they have to be regarded as less valid, due to partly small numbers of samples, as participants did not visit the different modules equally often. For this reason, the study phase analysis concentrates on the quantification over the geometric means of each participant's attention during his or her study phase and led to the general geometric means of Y1_study $=82.5 \%\left(\sigma_{\mathbf{Y} 1 \_ \text {study }}=\mathbf{1 . 4}\right)$ for the FocusMetaphor Interface and Y2_study $=\mathbf{7 0 . 0} \%\left(\boldsymbol{\sigma}_{\mathbf{Y} 2 \_ \text {study }}=\mathbf{3 . 2}\right)$ for the Grid Layout Interface. These findings are clearly higher than the results of the overall attention analysis (see comparison in fig. 3) which can be deduced by the elimination of interfering sequences of navigation from one module to another.

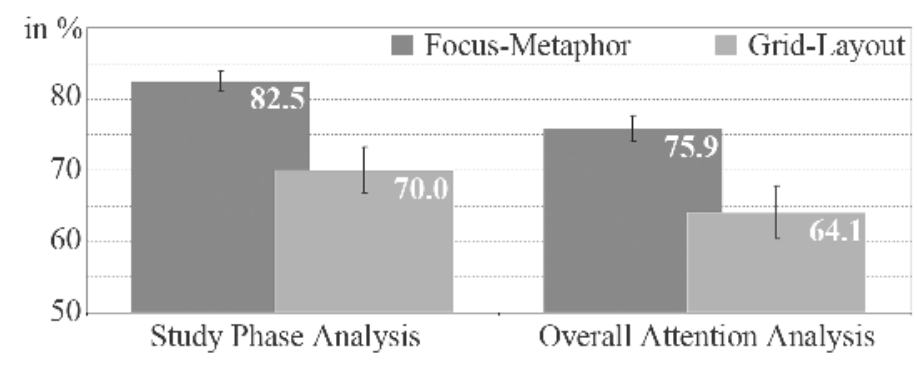

Fig. 3. Comparison of User attention

Nonetheless, the findings of the study phase analysis correspond to the ones of the overall attention analysis and therefore corroborate the first assumption of providing a better focus onto the learning context. In addition, this analysis points out the decreased disruption by navigational elements when using the Focus-Metaphor Interface to study information.

Table 3. Comparison of user attention

\begin{tabular}{c|ccc|ccc}
\hline & \multicolumn{3}{|c|}{ Focus-Metaphor } & \multicolumn{3}{c}{ Grid Layout } \\
& in \% & $\boldsymbol{\sigma}_{\mathbf{Y} 1}$ & Conf. Interval & in \% & $\boldsymbol{\sigma}_{\mathbf{Y} 2}$ & Conf. Interval \\
\hline $\begin{array}{c}\text { Study Phase } \\
\text { Analysis }\end{array}$ & 82,5 & 1,4 & $79,3 \leq \mu \leq 85,6$ & 70,0 & 3,2 & $63,1 \leq \mu \leq 76,9$ \\
$\begin{array}{c}\text { Overall Atten- } \\
\text { tion Analysis }\end{array}$ & 75,9 & 1.8 & $72,0 \leq \mu \leq 79,7$ & 64,1 & 3,7 & $56,0 \leq \mu \leq 72,2$ \\
\hline
\end{tabular}

\subsection{Pre-switch Analysis}

The second assumption of the hypothesis, "an increased orientation", has been investigated mainly through the pre-switch analysis. This analysis compared the participants' attention onto the primary focus module (again in X[1] and X[2]) in short time frames before the participant switches from one module to another. This switch is de- 
fined through initialization of a change for the primary focus module by clicking a module which at this point of time is in secondary focus. The pre-switch analysis uses the time frames: eight seconds, four seconds and two seconds before a switch to analyze the participants' decreasing attention on the primary focus module while looking for "new" information with an accuracy of at least 0.1 seconds.

As expected, both versions, X[1] and X[2], show a decrease of visual attention, the shorter the time frame gets. This reflects the efforts of the participants to 'scan' other modules for potentially interesting information, which logically increases, when the participants approach the moment where they finally switch the focus.

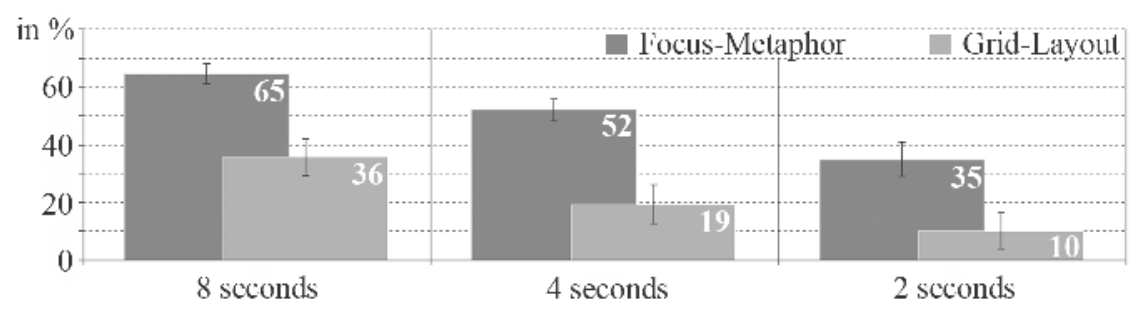

Fig. 4. User Attention before a switch of the primary focus

The comparison between X[1] and X[2] reveals, that the Focus-Metaphor Interface showed conspicuously higher values than the grid layout interface. Table 4 on the next page shows the geometric means of user attention for the specific time frames together with the according standard errors and confidence intervals. The results point out that participants needed clearly less time with the Focus-Metaphor Interface to decide on where they want to switch their focus to. As a result, they can spend more time onto the content they want to study. Moreover, it can be concluded that navigation decisions have been easier for participants with the Focus-Metaphor Interface. These aspects lead to an optimized orientation, as claimed in assumption two. Nevertheless it should be mentioned that participants switched content in the Focus-Metaphor scenario 7.1 times in average, and therefore slightly more often than in the grid layout scenario with 6 times over the length of the session.

Table 4. User attention before a focus switch

\begin{tabular}{c|ccc|ccc}
\hline \multirow{2}{*}{$\begin{array}{c}\text { Time before } \\
\text { a focus switch }\end{array}$} & \multicolumn{3}{|c|}{ Focus-Metaphor } & \multicolumn{3}{c}{ Grid Layout } \\
& in \% & $\boldsymbol{\sigma}_{\mathbf{Y} 1}$ & Conf. Interval & in \% & $\boldsymbol{\sigma}_{\mathbf{Y} 2}$ & Conf. Interval \\
\hline 8 seconds & 65 & 3.5 & $57,0 \leq \mu \leq 72,1$ & 36 & 6.4 & $22,1 \leq \mu \leq 49,5$ \\
4 seconds & 52 & 3.8 & $43,8 \leq \mu \leq 60,3$ & 19 & 6.6 & $5,0 \leq \mu \leq 33,6$ \\
2 seconds & 35 & 6 & $22,1 \leq \mu \leq 47,8$ & 10 & 6.4 & $-3,6 \leq \mu \leq 24,1$ \\
\hline
\end{tabular}

\section{Discussion}

The Focus-Metaphor Approach gives users an interface with clearly defined elements of information, which aims to match their cognitive capacity. Arranging these 
elements in a circular way around users' visual center and hosting the information they 'asked for' in this center, reflects their natural perception.

The conducted testing and evaluation of the Focus-Metaphor interface prototype tried to prove its usability through comparison with a standard design approach and assess general acceptance by the test participants. Both aspects are regarded as generally essential for the success of a novel approach towards user interface design. In the concrete case of the Focus-Metaphor approach, they have been investigated through an objective experiment and a subjective evaluation by potential users to decide on the worthiness of further work. The results of the online evaluation reveal the affection of participants towards the interface prototype. Moreover, the results of the eyetracking experiment are promising in that they show advantages for the FocusMetaphor approach in all measures. The calculation of confidence intervals adds some further significance to the results, but the limitations of the overall analysis should be kept in mind. As the sample sizes allowed only estimated measures, a second run of the eye-tracking experiment could add great value to the already gathered data.

In addition, it is the novelty of this approach, which needs careful consideration when evaluating the results. As Baudisch et al. [6] pointed out for their project: "A long term study is required to investigate whether users' subjective preference may reverse itself as users gain more experience with this still fairly uncommon visualization style."

Nevertheless the described work provides a detailed picture of the significance of the Focus-Metaphor approach, including the first usable implementation of a FocusMetaphor interface and testing results.

With regard to further work, the Focus-Metaphor interface prototype can be regarded as a very early stage of a framework which will allow the development of online environments, which are user-centric, adapted to cognitive processes and fully dynamic. Adding, removing or changing information or functionality will be easily possible through integration of new modules.

Moreover, a Focus-Metaphor framework could offer different visualisation modes according to users' preferences or device-side boundaries. Besides the centred animated one, used for this prototype, a further development of a manual mode, which has briefly been mentioned, seems to be of high potential and is definitely worth ongoing efforts.

The emerging research on mobile learning could likely profit from interface solutions which are applicable across platforms. Here, a framework solution of an adaptable and flexible user interface, which the Focus-Metaphor Approach is able to offer, could deliver additional value.

Within online environments, communities become increasingly important in form of communities of interest or communities of practice. As the core of a community is always to exchange information, collaborative information environments [24] for the Internet are actually of great interest. One big issue in the design of respective environments is the visualisation of collaboration, also referred to as community memories [24].

It has already been pointed out how the Focus-Metaphor Approach aims to model individual memories through flexible organisation of information, adaptive navigation and a visualisation which reflects the actual short-term memory. Under the 
assumption that this will work after spending a considerably large amount of effort on the further development of this approach, in a next step, which might be considerably small, visualizations of individual networks of knowledge could be incorporated into the visualization of a network of group knowledge.

\section{Acknowledgements}

This work was conducted by the first author as part of an MSc [25] with the second author as supervisor.

Further work on the Focus-Metaphor approach is planned and will most likely be conducted by the first author as part of a PhD at University College London.

\section{References}

1. Card, S. K. et al. (2000). Browse Hierarchical Data with the Degree of Interest Tree. Palo Alto Research Centre \& National Security Agency.

2. Card, S. K. \& Nation, D. (2002). Degree-of-Interest Trees: A Component of an AttentionReactive User Interface. Palo Alto Research Centre.

3. TheBrain Technologies Corporation, http://www.thebrain.com/

4. Gutwin, C. (2002). Improving Focus Targeting in Interactive Fisheye Views. Proceedings of the ACM Conference on Human Factors in Computing Systems (CHI'02), Minneapolis, pp. 267-274.

5. Thomas, C. (2002). Fisheye Strategy. Theories in Computer human interaction, University of Maryland, USA. http://www.cs.umd.edu/class/fall2002/cmsc838s/tichi/fisheye.html

6. Baudisch, P. et al. (2004). Fishnet, a fisheye web browser with search term popouts: a comparative evaluation with overview and linear view. Microsoft Research, USA.

7. ICQ Universe, http://www.icq.com

8. Demaine, J. (1996). Library and Archives Canada - Information Visualization http://www. collectionscanada.ca/

9. Hornof, A. J. (2002). Cognitive modelling, visual search, and eye tracking. ONR Attention, Perception and Data Visualization Workshop, George Mason University.

10. Wirth, T. (2002). Missing Links. Hanser publishing, Germany.

11. Fuster, J. M. (1998). Linkage at the Top. In: Neuron, Vol. 21, pp. 1223-1229.

12. Schulmeister, R. (2002). Grundlagen Hypermedialer Lernsysteme. Oldenbourg Verlag, Germany.

13. Kuhlen, R (1991). Hypertext, ein nicht-lineares Medium zwischen Buch und Wissensbank. Springer: Berlin.

14. Bruner, J. S. (1966) Toward a Theory of Instruction. Harvard University Press: Cambridge, Mass., USA.

15. Kolb, D. A. (1984). Experiential Learning: Experience as the source of learning and development. New Jersey: Prentice Hall.

16. Miller, G. A. (1956). The Magical Number Seven, Plus or Minus Two: Some Limits on our Capacity for Processing Information. In: Psychological Review, Vol. 63, pp. 81-97.

17. Pastor, M. - Short-Term Memory. San Diego State University http://coe.sdsu.edu/ eet/Articles/stmemory/start.htm

18. Laqua, S. (2003). Concept and user interface design for a cscl-environment for intercultural communication. Bachelor Dissertation at University of Technology, Dresden. 
19. MyNetscape, http://my.netscape.com/

20. MyMSN, http://my.msn.com/

21. O'Callahan, J. (2004). Crafting Personal Stories. Reprinted from Storytelling Magazine, May/June 2004. On: www.ocallahan.com

22. Perlman, G. (1998). Web-Based User Interface Evaluation with Questionnaires. http://www.acm.org/ perlman/question.html

23. Lewis, J. R. (1995). IBM Computer Usability Satisfaction Questionnaires: Psychometric Evaluation and Instructions for Use. In: International Journal of Human-Computer Interaction, Vol. 7, pp. 57-78.

24. Stahl, G. (2004) "Collaborating with Technology: Studies in Design \& Theory of Online Collaboration". Book preprint from MIT Press.

25. Laqua, S. (2004). "Creation of Virtual Social Networks in Distanced, Informal Learning Settings through collaborative Story Writing - Implementation and Testing of a New Metaphor Prototype". Master Dissertation at Northumbria University in Newcastle. 